\title{
El perfil emprendedor tras el fracaso
}

\author{
Jorge Rodrigo Espinoza-Benavides ${ }^{\mathrm{a}, 1}$, David Díaz \\ ${ }^{a}$ Universidad Católica de la Santísima Concepción, Concepción, Chile \\ ${ }^{b}$ Universidad de Chile, Santiago, Chile
}

doi: $10.20420 /$ eni.2020.327

\begin{abstract}
Resumen
El objetivo de éste artículo es examinar el perfil de los emprendedores que fracasaron en negocios anteriores, pero que a pesar de su fracaso inicial, decidieron iniciar o mantener otro negocio; en el contexto de un país latinoamericano (Chile). Los resultados muestran que para los emprendedores chilenos, hay un perfil diferente entre los que emprenden por primera vez (novatos) y los que deciden iniciar un nuevo negocio después de fracasar. Estas diferencias muestran que los emprendedores que han fracasado e iniciado un nuevo negocio se comportan como inversores ángeles, tienen mejores habilidades empresariales y tienen más experiencia. Además, es más probable que compartan la propiedad de su nuevo negocio.
\end{abstract}

Palabras clave: salida, perfil del emprendedor, dimensiones institucionales, árboles de decisión.

Clasificación JEL: L26, O54.

Fuente de referencia: Espinoza-Benavides, J., \& Díaz, D. (2019). The entrepreneurial profile after failure. International Journal of Entrepreneurial Behavior \& Research, IJEBR-04-2018-0242. https://doi.org/10.1108/IJEBR-04-2018-0242.

\section{Introducción}

El proceso de entrada y salida de empresas de un mercado es clave para la productividad de las economías nacionales. Como tal, este proceso ha sido ampliamente estudiado en la literatura y es bien sabido que puede ser determinado por factores específicos de la empresa, la industria y el país (Carree \& Thurik, 1996). Por el contrario, el proceso por el cual los individuos deciden entrar y salir de un emprendimiento de negocio es menos conocido. En este sentido, existen preguntas abiertas referentes a los efectos potenciales que la salida o el fracaso de una empresa pueden generar en un emprendedor. Siguiendo a Hessels, Grilo y Thurik (2011), basado en la teoría del comportamiento humano de Becker, este trabajo considera la salida empresarial como un indicador del capital humano empresarial acumulado, que se refleja en la adquisición de conocimientos, habilidades y experiencia. Hessels y otros (2011) muestra que el fracaso genera un mayor compromiso empresarial, especialmente para los hombres, para las personas que conocen a otro empresario y para aquellos que tienen un bajo nivel de miedo al fracaso. Sobre la base de estos resultados, es posible cuestionarse si los emprendedores que han fracasado anteriormente son realmente diferentes de los emprendedores que no han fracasado. Además, si estos hallazgos reflejan el caso general o si es posible encontrar grupos heterogéneos que tienen una relación diferente con el fracaso. En este estudio la teoría institucional (North, 1990) se utiliza, también, como marco que podría ayudar a explicar las variables que determinan los diferentes perfiles de los emprendedores, sobre todo aquellos aspectos que conforman el marco regulatorio (instituciones), formal en informal, de una determinada economía o país (North, 1990).

El marco, a su vez, ayuda a justificar por qué tiene sentido centrarse en un país para estudiar la existencia potencial de estos diferentes grupos. Así, dado que las características de las instituciones formales e informales pueden llevar a la creación de clústeres de emprendedores, los perfiles podrían ser más difíciles de aislar en un análisis que incluye a varios países, como el que realizaron Hessels y otros (2011).

${ }^{1}$ Autor de correspondencia: jespinoza@ucsc.cl 
Chile es un país que presenta una oportunidad adecuada para caracterizar la existencia de diferentes perfiles de emprendedores, a partir del fracaso, por varias razones. Chile, país latinoamericano, se encuentra en la etapa de transición de una economía emergente a una economía desarrollada, al mismo tiempo, Chile tiene una de las peores distribuciones de ingresos de la OCDE, contexto en que el emprendimiento juega un papel importante en el crecimiento, lo que conlleva un fuerte contexto público $\mathrm{y}$ privado de fomento a la creación de nuevos negocios.

Como tal, entonces el objetivo principal del estudio es caracterizar el perfil de los emprendedores que han fracasado en negocios anteriores, pero que, a pesar de su fracaso inicial, deciden iniciar un nuevo negocio en Chile. Este tipo de emprendedores, los denominamos "reemprendedores después del fracaso empresarial".

El trabajo contribuye con resultados empíricos que validan que el fracaso conduce a características especiales en los emprendedores, empleando una metodología denominada árboles de decisión, que es relativamente nueva en la comunidad de estudio sobre el fenómeno del emprendimiento. El trabajo también discute cómo las instituciones podrían desempeñar un papel en este fenómeno.

Una vez que se identificaron las dimensiones teóricas que podrían influir en la actividad emprendedora, en base a varios estudios previos analizados (Byrne, Mullen, \& Marlow, 2011; Hessels y otros, 2011; Olugbola, 2017; Shepherd \& Kuratko, 2009; Stam, Audretsch, \& Meijaard, 2008; Ucbasaran, Shepherd, Lockett, \& Lyon, 2013) los autores propusieron un conjunto de cinco hipótesis de estudio.

\section{Sobre los Métodos de Análisis Empleados}

Para estudiar las hipótesis, se empleó la información del Global Entrepreneurship
Monitor (GEM, Adult Population Survey 2016 para Chile). A partir de las respuestas de la encuesta, se definen dos grupos de emprendedores, "re-emprendedores" y "emprendedores por primera vez (o novatos)"; los primeros son aquellos que han fracasado y han decidido volver a crear una nueva empresa, y los segundos son aquellos que recién se inician en el mundo empresarial (y que obviamente no han fracasado aún).

El conjunto de datos original consta de 9.196 registros, dentro de los cuales se identificaron 2.024 registros de individuos participando, respectivamente, en etapas tempranas de un emprendimiento; a su vez, dentro de este último grupo se identificaron 233 casos de reemprendedores Entonces los análisis, que se describen brevemente a continuación, se concentraron en comparar al grupo de 233 individuos con el grupo de emprendedores (2.024), restando al número de re-emprendedores $(2.024-233=1.721)$, vale decir la cuantía de emprendedores novatos.

Inicialmente, se aplica un análisis de ANOVA para estudiar cómo varias características individuales y contextuales se relacionan con la pertenencia a uno de estos grupos. Posteriormente, se utiliza una regresión logística como prueba multivariante de la relación entre esta variable dicotómica dependiente y varias variables explicativas.

En una segunda etapa, se propone una herramienta novedosa para presentar en detalle los patrones que se asocian con cada grupo. Un algoritmo de árbol de decisión (Kohavi \& Quinlan, 2002) encuentra y describe patrones lineales y no lineales disponibles en los conjuntos de datos como una colección de "ifthen-rules (reglas: si-entonces)" que también se expresan gráficamente como un gráfico de decisión. El análisis de estos patrones nos ayuda a descubrir nuevas perspectivas con respecto a las características de cada grupo. 


\section{Principales Resultados y Conclusiones}

Tanto el análisis de ANOVA como la regresión logística comprueban la mayoría de las hipótesis planteadas, permitiendo afirmar que el perfil del grupo de re-emprendedores se caracteriza por ser más propenso a asumir el papel de inversionista ángel ${ }^{2}$, a tener una clara identificación como propietario de su negocio, a tener más habilidades y un nivel educativo más bajo en comparación con los empresarios noveles (novatos).

Considerando, también, tanto los resultados del ANOVA como de la regresión logística, la voluntad de invertir como inversor ángel y la forma en que se estructura la propiedad de la empresa son inicialmente las variables más significativas que permiten diferenciar a los grupos de nuevos emprendedores y reemprendedores.

Estos resultados están directamente relacionados con lo que plantearon varios autores (Carter \& Wilton, 2006; Cardon, Stevens \& Potter, 2011) con respecto a los efectos de los marcos regulatorios en materia financiera y fiscal (impuestos) y su capacidad para fomentar $\mathrm{u}$ obstaculizar el espíritu empresarial. El perfil de los emprendedores en situación de post-fracaso, tanto en su propensión a ser inversores ángeles como en la participación en la propiedad de sus empresas, también puede estar relacionado con las instituciones reguladoras. Ambas interpretaciones reflejan la importancia del contexto y de las instituciones, ya sean formales o informales, y por lo tanto proporcionan evidencia para apoyar la incorporación de la teoría institucional (North, 1990) en el marco del análisis.
El resultado del procedimiento de selección de características fue plasmado en el árbol de decisión $^{3}$ (ver figura 1), es decir, la importancia de los predictores. En primer lugar, es posible apreciar que las características individuales, como el nivel de educación, son muy importantes para discriminar entre grupos de reemprendedores y emprendedores novatos. En orden descendente, otras variables importantes son si el emprendedor será propietario de una parte de su negocio o del total, si el miedo al fracaso le impedirá iniciar un negocio y si ha actuado como inversionista ángel. Entonces estas variables se configuran de tal manera que encontramos cuatro grupos de re-emprendedores y cinco grupos diferentes de emprendedores novatos (ver figura 1)

Al interpretar algunas reglas del árbol de decisiones (figura 1 , regla $3 /$ rule 3 ; para reemprendedores/ re-entrepreneurs), como un clúster o sub-segmento de la población, es posible corroborar los resultados del modelo logístico porque describe un subconjunto de emprendedores (que han fracasado) cuyas características son las siguientes: poseen sólo una parte de sus negocios (variable: suown, en figura 1), no han alcanzado un alto nivel educativo (variable: EDUC_LEVEL, en figura 1) y tienden a actuar como inversores ángeles (variable: busang, en figura 1$)^{4}$.

Aunque, desde el punto de vista estadístico, es posible estar seguro de que existen estos cuatro subgrupos de re-emprendedores, cuando se comparan los conjuntos de normas con las normas para los emprendedores novatos, las diferencias no son tan evidentes para el ojo humano. Los patrones más interesantes son que un subgrupo de re-emprendedores (regla 1/rule 1, panel izquierdo, figura 1) es casi idéntico a un subgrupo de los emprendedores novatos (regla

\footnotetext{
${ }^{2}$ Un inversionista ángel es una persona que aporta capital a un emprendimiento en fase temprana, transformándose en socio capitalista. Estos inversionistas no necesariamente tienen experiencia previa en negocios o realizando inversiones. Es posible que formen parte de la red de amigos y/o familiares del emprendedor.

${ }^{3}$ La figura 1 está en idioma inglés, pues el software empleado arroja los resultados en ese idioma y no otorga la posibilidad de edición.

${ }^{4}$ El resto de las variables abreviadas del inglés, en la figura 1 , significan: suskill = habilidades para emprender y opport $=$ detección de oportunidades.
} 
3/rule 3, panel derecho, figura 1). Estos dos subgrupos comparten la mayor parte de sus características excepto por el hecho de que el nivel educativo en el subgrupo de reemprendedores es más bajo, incorporando a personas con 10 años de educación. Además, hay un gran subgrupo de emprendedores novatos que no actúan como ángeles inversores (regla 4, panel derecho, figura 1).

Aunque no existe un acuerdo completo en cuanto a las características identificadas por Hessels y otros (2011), es posible afirmar que ser un ángel inversor es una característica diferenciadora entre los re-emprendedores y los emprendedores novatos. Esta evidencia muestra un vínculo potencial entre la teoría de los inversionistas ángeles y la teoría del comportamiento emprendedor, ya que el perfil de los inversionistas ángeles no se caracteriza por el espíritu emprendedor, según Morrissette (2007). Sin embargo, estudios recientes permiten corroborar que la experiencia previa, como empresario, influye en las decisiones de los inversores ángeles (Harrison, Botelho \& Mason, 2016).

Así, la principal conclusión del estudio es que existe un perfil empresarial diferente entre los empresarios que fracasan (re-emprendedores) y los que no han fracasado recientemente (empresarios novatos) en Chile. Este perfil se define principalmente asumiendo el rol de inversores ángeles, compartiendo la propiedad de su negocio, teniendo habilidades empresariales y niveles de educación relativamente más bajos. Estas cuatro variables han sido validadas por los tres métodos que se aplicaron en nuestro estudio. Esta constatación contribuye a reforzar la importancia del fracaso en el proceso empresarial, tanto en lo que se refiere a las consecuencias positivas como negativas para las personas. Esto también está en línea con varios estudios previos sobre el tema, principalmente en países que no forman parte de América Latina (Hessels y otros, 2011).

Figura 1. Conjunto de reglas del árbol de decisión

\begin{tabular}{|c|c|}
\hline 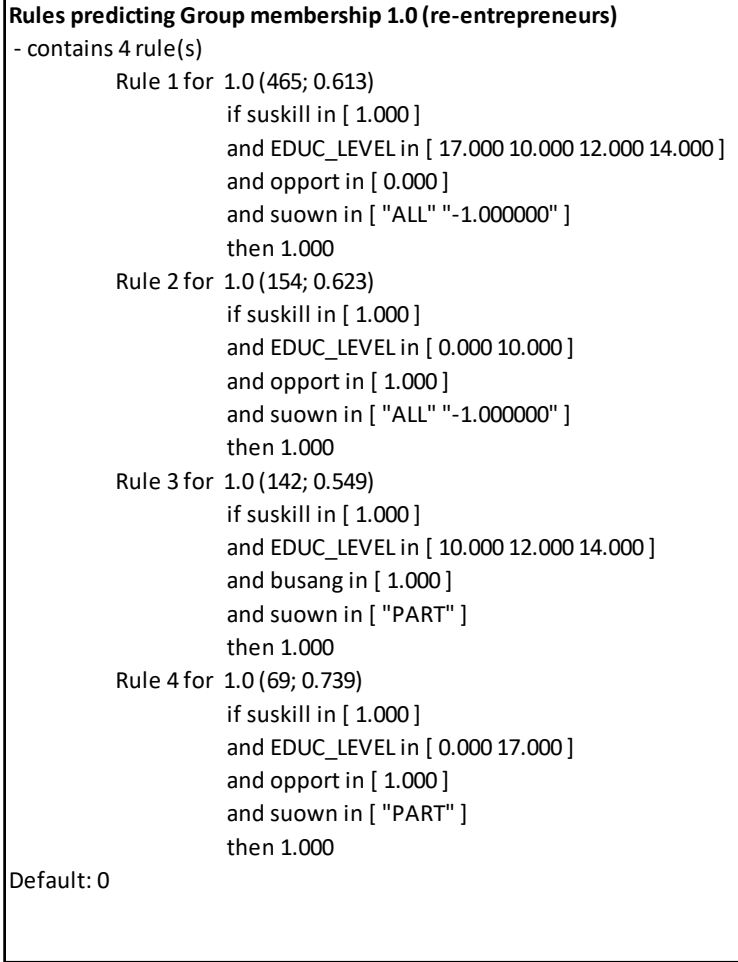 & 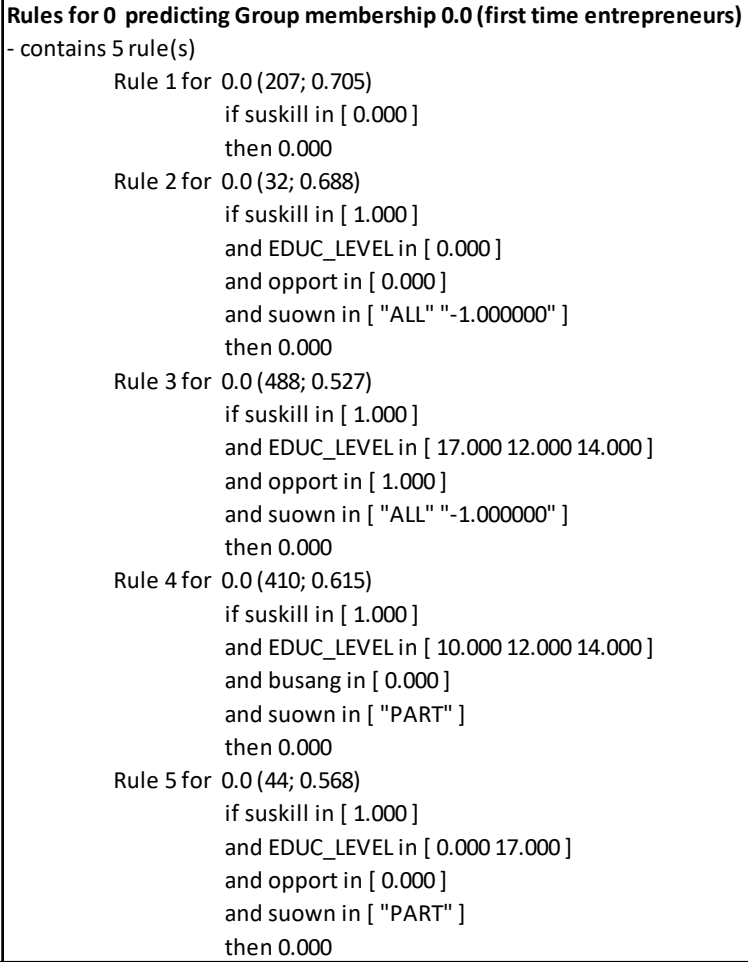 \\
\hline
\end{tabular}


En esta línea, la investigación es relevante porque, en países como Chile, las políticas públicas suelen estar orientadas a promover el emprendimiento por primera vez. Por otro lado, no hay apoyo para quienes han fracasado como empresarios; de hecho, las instituciones formales e informales pueden obstaculizar la posibilidad de crear un nuevo negocio en lugar de facilitarlo. Además, teóricamente, se puede argumentar que los empresarios que han fracasado tienen un mayor potencial de impacto económico comparado con los empresarios que no han fracasado y que están iniciando su primer negocio. Por estas razones, el propósito de obtener información sobre los perfiles de los empresarios que, a pesar del fracaso, han vuelto a empezar puede ser utilizado por las autoridades y las comunidades académicas para comprender y diseñar mejor las políticas que podrían tener un mayor impacto sobre estos temas.

Para conocer mayor detalle de los resultados y profundizar en las conclusiones del estudio puede revisar el trabajo de Espinoza-Benavides y Díaz (2019).

\section{Referencias}

Byrne, O., Mullen, H., \& Marlow, S.M.C. (2011). Repeat Entrepreneurship following Business Closure. Glasgow, Scotland.

Cardon, M.S., Stevens, C.E. \& Potter, D.R. (2011). Misfortunes or mistakes? Cultural sensemaking of entrepreneurial failure. Journal of Business Venturing, 26(1), 79-92.

Carree, M., \& Thurik, R. (1996) 'Entry and exit in retailing: Incentives, barriers, displacement and replacement'. Review of Industrial Organization, 11(2), 155-172.
Carter, S., \& Wilton, W. (2006). Don't Blame The Etrepreneur, Blame Government: The Centrality of The Gorvenment in Enterprise Development; Lessons from Enterprise Failure in Zimbabwe. Journal of Enterprising Culture, 14(01), 65-84.

Espinoza-Benavides, J., \& Díaz, D. (2019). The entrepreneurial profile after failure. International Journal of Entrepreneurial Behavior \& Research, $25(8), 1.634-1.651$.

Harrison, R.T., Botelho, T., \& Mason, C.M. (2016). Patient capital in entrepreneurial finance: a reassessment of the role of business angel investors, Socio-Economic Review, 14(4), 669-689.

Hessels, J., Grilo, I., Thurik, R., \& van der Zwan, P. (2011). Entrepreneurial exit and entrepreneurial engagement. Journal of Evolutionary Economics, 21(3), 447-471.

Kohavi, R., \& Quinlan, J.R. (2002). Data mining tasks and methods: Classification: decision-tree discovery. Handbook of Data Mining and Knowledge Discovery. Oxford University Press.

Morrissette, S.G. (2007). A Profile of Angel Investors. The Journal of Private Equity, 52-66.

North, D.C. (1990). Institutions, Institutional Change and Economic Performance. Cambridge University Press. Cambridge.

Olugbola, S.A. (2017). Exploring entrepreneurial readiness of youth and startup success components: Entrepreneurship training as a moderator. Journal of Innovation \& Knowledge, 2(3), 155-171.

Shepherd, D.A., \& Kuratko, D.F. (2009). The death of an innovative project: How grief recovery enhances learning. Business Horizons, 52(5), 451-458.

Stam, E., Audretsch, D., \& Meijaard, J. (2008). Renascent entrepreneurship. Journal of Evolutionary Economics. Springer-Verlag, 18(3-4), 493-507.

Ucbasaran, D., Shepherd, D.A., Lockett, A., \& Lyon, S.J. (2013). Life after business failure: the processand consequences of business failure for entrepreneurs. Journal of Management, 39(1), 163-202. 